\section{APPROPRIATENESS OF PRESCRIBING OF DIRECT ORAL ANTICOAGULANTS IN A UNIVERSITY TEACHING HOSPITAL NETWORK}

${ }^{1}$ Francis Elechi ${ }^{*},{ }^{2}$ Sotiris Antoniou, ${ }^{3}$ Vikas Kapil. ${ }^{1}$ Barts and the London School of Medicine and Dentistry; ${ }^{2}$ Cardiac Research Centre, St Bartholomew's Hospital, London; ${ }^{3}$ William Harvey Research Institute, QMUL

\subsection{6/heartjnl-2019-BCS.116}

Introduction Direct oral anti-coagulants (DOACs) have been developed that provide simplified, fixed dose regimes over variable dosing with vitamin $\mathrm{K}$ anticoagulants. However, DOAC dosing is dependent on indication, demographics and co-morbidities, such as renal impairment. Inappropriate dosing is associated with harm with both unintentional under- and excessive-dosing [1]. The purpose of this study is to describe the appropriateness of DOAC prescribing with respect to the criteria set out in summary of product characteristics (sPC) and British National Formulary [2,3].

Methods A retrospective audit of consecutive in-patients, over a 3-month period, prescribed DOACs in St Bartholomew's and Royal London hospitals from November 2018 was performed. Data was extracted from electronic healthcare and pharmacy records. Inclusion criteria: prescribed a DOAC (apixaban, dabigatran, edoxaban and rivaroxaban), recorded dose and frequency, patient sex, age and weight; serum creatinine $(\mathrm{sCr})$. Exclusion criteria: use of non-DOAC anticoagulant, record duplication and incomplete records. Dosing appropriateness was compared for each of the drugs with respect to indication and criteria set in individual sPCs.

Results 360 patients were prescribed DOACs, of whom 203 $(56 \%)$ satisfied inclusion/exclusion criteria. Main reasons for non-inclusion were incomplete records $(n=54)$ and record duplication $(n=91)$. Mean age was $76 \pm 24$ years, with $23 \%$ of patients $>80$ years-old and $15 \%<60 \mathrm{~kg}$. Renal function (creatinine clearance $(\mathrm{CrCl})$, calculated using Cockcroft-Gault method) was $<50 \mathrm{~mL} / \mathrm{min}$ in $42 \%$ of patients. The majority of DOAC prescribing was for atrial fibrillation (71\%). For the individual DOACs, dose appropriateness was best for dabigatran (93.3\%, $\mathrm{n}=14 / 15$ patients) and edoxaban (95.2\%, 20/21 patients). Rivaroxaban was prescribed appropriately in $81 \%$ $(\mathrm{n}=58 / 72)$ of patients and apixaban was prescribed appropriately in $82 \%(n=78 / 95)$ of patients. Majority of dose inappropriateness was due to non-indicated underdosing $(n=29 /$ 34). For rivaroxaban, this was apparent in patients with a significant discrepancy between renal function measured by eGFR (CKD-EPI equation) and calculated $\mathrm{CrCl}$ (CockroftGault method, recommended in sPC and used in the clinical trials). For patients requiring reduced dosage, this was correctly prescribed in 92\% $(n=35 / 38)$ patients.

Conclusion In our university hospital network, more patients were underdosed relative to their indication than was expected. This may be due to usage of clinically available eGFR, which is routinely available as part of hospital systems, rather than using manually calculated $\mathrm{CrCl}$ and the multiple criteria required to dose apixaban correctly. Further education of prescribers and ward-based pharmacists is required to improve the prescribing quality with the aim of improving treatment efficacy and safety outcomes.

\section{REFERENCES}

1. Yao X, et al. J Am Coll Cardiol 2017:69:2780.

2. www.medicines.org.uk

3. www.bnf.nice.org.uk

\section{Conflict of Interest nil}

\section{CARDIOVASCULAR RISK PROFILE AND DISEASES IN PATIENTS WITH SARCOIDOSIS IN THE ACALM BIG DATA REGISTRY}

${ }^{1}$ Satpal Ubhi*, ${ }^{2}$ Paul Carter, ${ }^{3}$ Chris Miller, ${ }^{4}$ Ranjit More, ${ }^{5}$ Shajil Chalil, ${ }^{5}$ Rahul Potluri. ${ }^{1}$ Cambridge University Hospitals NHS Foundation Trust; ${ }^{2}$ ACALM Study Unit; ${ }^{3}$ Manchester Foundation Trust; ${ }^{4}$ Blackpool Teaching Hospital NHS Foundation Trust; ${ }^{5}$ Blackpool Teaching Hospitals

10.1136/heartjnl-2019-BCS.117

Abstract 120 Table 1 Prevalence of comorbid cardiovascular disease in patients with sarcoidosis

\begin{tabular}{|l|ccc|}
\hline & Sarcoid Prevalence & Control Prevalence & Adjusted Risk \\
& $\mathrm{n}(\%)$ & $\mathrm{n}(\%)$ & Odds ratio $(95 \% \mathrm{CI})$ \\
\hline Hypertension & $256(28.4 \%)$ & $1,562(17.3 \%)$ & $1.724(1.430-2.078)^{* * *}$ \\
Hyperlipidemia & $102(11.3 \%)$ & $605(6.7 \%)$ & $1.310(1.014-1.691)^{*}$ \\
T1DM & $19(2.1 \%)$ & $107(1.2 \%)$ & $1.284(0.769-2.144)$ \\
T2DM & $152(16.9 \%)$ & $682(7.6 \%)$ & $1.961(1.587-2.425)^{* * *}$ \\
IHD & $97(10.8 \%)$ & $801(8.9 \%)$ & $0.849(0.656-1.097)$ \\
ACS & $13(1.4 \%)$ & $242(2.7 \%)$ & $0.394(0.220-0.707)^{* *}$ \\
Heart Failure & $51(5.7 \%)$ & $209(2.3 \%)$ & $2.202(1.549-3.130)^{* * *}$ \\
AF & $46(5.1 \%)$ & $283(3.1 \%)$ & $1.215(0.855-1.727)$ \\
CKD & $43(4.8 \%)$ & $122(1.4 \%)$ & $2.862(1.967-4.164)^{* * *}$ \\
Cerebrovascular & $17(1.9 \%)$ & $156(1.7 \%)$ & $0.814(0.482-1.375)$ \\
PVD & $8(0.9 \%)$ & $104(1.2 \%)$ & $0.473(0.221-1.010)$ \\
\hline
\end{tabular}

Odds ratio for comorbid cardiovascular disease adjusted by multivariate logistic regression accounting for demographics and cardiovascular diseases ${ }^{*}=\mathrm{p}<0.05{ }^{* *}=\mathrm{p}<0.01{ }^{* * *}=\mathrm{p}<0.001$ T1DM= Type 1 Diabetes Mellitus. T2DM= Type 2 Diabetes Mellitus. IHD= Ischemic Heart Disease. ACS= Acute Coronary Syndrome.

$\mathrm{AF}=$ Atrial Fibrillation. $\mathrm{CKD}=$ Chronic Kidney Disease. $\mathrm{PVD}=$ Peripheral Vascular Disease. 